\title{
Fruits \& vegetables are the actual fast foods
}

\begin{abstract}
The burden of non-communicable diseases has been rapidly increasing all around the world. In year 2001 non- communicable disease accounted for 60 percent of the 56 million deaths worldwide and 47 percent of the global burden of disease. Underlying determinants for non-communicable diseases includes increased consumption of energy-dense, nutrient-poor foods that are high in fat, sugar and salt; reduced levels of physical activity: and of particular concern are the increasingly unhealthy diets and reduced physical activity of children and adolescents. Decreasing the prevalence of non-communicable diseases is therefore still an important goal in the public health strategy. For this purpose, consumption of fruit and vegetables is generally considered to be beneficial to health, and there is now a comprehensive body of evidence recognizing the protective effects of high fruit and vegetable consumption on cardiovascular disease and certain cancers, particularly epithelial cancers. Fruits \& vegetables should be called as fast food. They can be consumed fresh, cooked, hot or cold, canned, pickled, frozen or dried. Fruits and vegetables are consumed at all times, and due to their convenient size; they are an excellent between-meal snack. They are relatively low in calories and fat (avocado and olives being the exceptions), they have no cholesterol, they are rich in carbohydrates and fiber, they contain vitamin $\mathrm{C}$ and carotene, and some are a good source of vitamin B complex. Because of all these characteristics, fruits and vegetables have a unique role in a healthy diet
\end{abstract}

Keywords: fruits, vegetables, non-communicable diseases, fast food
Volume 6 Issue 3 - 2017

\section{Syeda Aima Bokhari}

Food Science \& Human Nutrition Department, University of Veterinary \& Animal Sciences, Pakistan

Correspondence: Syeda Aima Bokhari, University of Veterinary \& Animal Sciences, Food Science \& Human Nutrition Department, Outfall road Lahore, Pakistan,

Email syedaaimabokhari@gmail.com

Received: February 02, 2017 | Published: March 02, 2017

\section{Introduction}

It's a popular saying "Eat healthy \& stay healthy". But, unfortunately now-a-days, the food consumed by most of people has great health hazards. With changes in lifestyle concepts \& relationships no one has enough time to think that what's good for the health \& what's not. With increasing globalization \& urbanization, people have started consuming high calorie foods known as fast food. Many health hazards have been related on consumption of such foods. Through health education, good eating practices can be adapted. ${ }^{1}$ Eating well helps people stay healthy and active and thus improves the quality of life. Good diet and eating habits are fundamental for proper growth and development and for the prevention of disease. ${ }^{2}$

The food that we eat is not only a source of providing nutrients for sustenance of life but it also contains a number of bioactive components that plays an important role in disease prevention and promotion of better health. Food pyramid contains a wide array of food groups in which the most important ones with a wide variety of colors and micronutrients are fruits $\&$ vegetables. There is a strong association in reducing the risk of cardiovascular diseases (CVD's), diabetes, cancer, Alzheimer disease \& cataracts through fruit \& vegetable consumption according to one's RDA. ${ }^{3}$

\section{In plant taxonomy fruits $\&$ vegetables are defined as}

The botanical term 'vegetables' refers to the edible part of a plant. Thus, fruits are actually a subclass of vegetables. The botanical term 'fruit' refers to the ripened ovary of a flower that contains seeds. This implies that plant foods, such as cereals, legumes, nuts, cucumbers and tomatoes, are all fruits. All other parts of the plants, such as stems, roots, and leaves, can be biologically considered as vegetables. ${ }^{4}$

Fruits \& vegetables are edible plant products with a great source of micronutrients and bioactive components required for good health.
That's why they are called nutrient rich foods. From the last decade, healthy life style is been given an immense amount of importance. To attain a healthy life style, a healthy diet is required to combat with the prevailing chronic diseases and for this purpose consuming a diet high in fruits and vegetables is receiving great amount of attention around the world. Both of these plant products are excellent sources of fiber, complex carbohydrates and numerous vitamins and minerals. Fruits and vegetables provide varying amounts and types of nutrients, therefore variety is essential. Thus the recommendation for daily fruit $\&$ vegetable consumption is three to five portions a day. ${ }^{5}$ Owing to the health benefits \& all the nutritional significance, fruits \& vegetables should be recommended as actual fast foods because they are convenient - handy size, can be eaten fresh, can be eaten on the go - can be taken as a snack, can be prepared quickly - wash and eat or peel and eat, provide quick energy - carbohydrates for our bodies, can be prepared quickly in a pan (stir-fried) or microwave (steamed). ${ }^{6}$

\section{Review of literature}

As it is usually said that "We are what we eat". With the passage of time, there is great awareness in people in order to live a healthy life. Healthy life is only attainable with the help of a balanced diet with variety of nutrients and physical activity. For this purpose, fruits and vegetables is a great source of variety of nutrients. There is a great number of evidence through different researches that regular intake of fruits \& vegetables in proposed amounts can be very helpful in preventing chronic disease which is major public health issue nowa-days. ${ }^{7}$

\section{In plant taxonomy fruits $\&$ vegetables are defined as}

The botanical term 'vegetables' refers to the edible part of a plant. Thus, fruits are actually a subclass of vegetables. The botanical term 'fruit' refers to the ripened ovary of a flower that contains seeds. This 
implies that plant foods, such as cereals, legumes, nuts, cucumbers and tomatoes, are all fruits. All other parts of the plants, such as stems, roots, and leaves, can be biologically considered as vegetables. ${ }^{4}$

Fruits and vegetables, both, provide a number of important nutrients including vitamins and minerals, fiber and a variety of phytochemicals. Both are low energy dense foods that can play an important role in managing body weight. For this purpose, an individual requires to replace high energy foods such as fatty foods with nutrient rich foods like fruits and vegetables. Fruits and veggies can also help in lowering the risk of heart diseases, increased blood pressure, diabetes \& cancer. The importance of fruits and vegetables as part of healthy diets is illustrated by the Dietary Guidelines for Americans 2010, in which two of the four recommended food groups are fruits and vegetables.

Low fruit and vegetable intake throughout the world has resulted in approximately 16million DALY's (disability adjusted lifeyears) (a measure of the potential life lost due to premature mortality and theyears of productive life lost due to disability) and mortality rate of around 1.7 million (2.8\%). Moreover, $14 \%$ of gastrointestinal cancer death, $11 \%$ of ischemic heart disease deaths and about $9 \%$ of stroke deaths worldwide are attributable to insufficient fruit \& vegetable intake. According to a recently published WHO/FAO report, a minimum intake of $400 \mathrm{~g}$ of fruit and vegetables per day (excluding potatoes and other starchy tubers) is recommended for the prevention of chronic diseases, as well as for the prevention and alleviation of several micronutrient deficiencies, especially in less developed countries.

\section{Types of fruit and vegetables}

The term "fruit and vegetables" encompasses a huge array of food products. From a botanical perspective we can sort vegetables into eight categories:

1. Root vegetables (e.g. carrots),

2. Tubers (e.g. potatoes),

3. Leafy (e.g. spinach),

4. Inflorescence or flower heads (e.g. broccoli),

5. Seed (e.g. green pea), bulbs (e.g. onions),

6. Stem or stalk (e.g. celery), and

7. Fruit vegetables (e.g. tomato, peppers).

Fruits can be sorted into

i. Citrus fruits (e.g. orange),

ii. Stone fruits (e.g. apricot),

iii. Pomme fruits (e.g. apples),

iv. Tropical/subtropical fruits (e.g. banana), and

v. Wild fruits (e.g. elderberry).

Along with the essential nutrition provided by fruit and vegetables, a variety of sensory properties are also given to the meals eaten. Fruits and vegetables help in enhancing presentation of a meal. In addition to their unique nutritive properties, adding a variety of rich colours can make a meal look more attractive. Fruit and vegetables also add texture, such as the crispy lettuce leaves in a salad or crunchy carrots in a spaghetti bolognese sauce. When vegetables are cooked, their texture changes as they soften. If we over-cook them, we lose the crisper texture and many of the good nutrients so it is better not to over-cook. ${ }^{4}$

\section{Colors of fruits $\&$ vegetables}

Fruit and vegetables are an important part of a good diet. In order to attain the maximum health benefits from fruit and vegetables, variety as well as quantity, both are extremely important because all the nutrients needed cannot be fulfilled by just one fruit or vegetable. Different colours of fruit and vegetables contain unique and specific combinations of nutrients and phytochemicals.

\section{Red fruit and vegetables (contain lycopene or anthocyanin)}

a. Cherry, strawberry, red grape, watermelon, pink grapefruit

b. Tomato, beetroot, red pepper

\section{Orange/Yellow fruit and vegetables (contain carotenoids)}

a. Orange, mango, pineapple, papaya, melon, lemon

b. Carrot, yellow tomato, yellow maize, yellow pepper, pumpkin, sweet potato

\section{Green fruit and vegetables (contain chlorophyll)}

a. Kiwifruit, green grape, avocado, lime

b. Kale, Chinese cabbage, spinach, broccoli, lettuce, cucumber, celery, green pepper, green pea

\section{Purple/ Blue fruit and vegetables (contain anthocyanin)}

a. Blueberry, black current, plum, purple fig

b. Eggplant, purple onion, purple cabbage, black olive

\section{White fruit and vegetables (contain anthoxanthins)}

a. Banana, pear, white peach

b. Cauliflower, cabbage, potato, white turnip, bean sprout, mushroom, onion, garlic, leek. ${ }^{8}$

\section{Nutritional Quality of Fruits \& Vegetables}

\section{Traditional components}

Water: Water is the most important single component of fruits and vegetables found in great amount, which is about $90 \%$ of the whole mass. The total amount of water in different fruits and vegetables may vary depending upon their structural differences.

Organic acids: The role of organic acids in fruits and vegetable is of great importance as it affects the flavour of these plant products by controlling the sugar to acid ratio. Two major types of acids found in fruits and vegetables are aliphatic (straight chain) and aromatic acids. Citric and malic acid (found in oranges and apples) are both aliphatic in nature and are found in abundance in fruits and vegetables. Aromatic organic acids are also found in several fruits and vegetables, but their concentration is quite low. Examples of aromatic acid include benzoic acid found in cranberries, quinic acid in bananas and chlorogenic acid in potatoes.

Proteins: Proteins represent less than $1 \%$ of the fresh mass of fruit and vegetable tissues. Leguminous seeds are rich in protein, 
containing $15 \%$ to $30 \%$. The proteins of fruits and vegetables are built from amino acids, but other related simple nitrogenous compounds also occur. Fruits are low in proteins, but tree nuts are a good source of high-quality proteins.

Lipids and fatty acids: Plant lipids represent a very broad group of compounds with a variety of functions. The fat content of fruits and vegetables is usually less than $1 \%$ and varies with the product. Examples of fat content on a dry mass basis are:
A. Avocado: $35-70 \%$
B. Olive: $30-70 \%$
C. Grape: $0.2 \%$
D. Banana: $0.1 \%$
E. Apple: $0.06 \%$

Fatty acids are aliphatic monocarboxylic acids that may be saturated or unsaturated. No double bond is present in saturated fatty acids along the chain while monounsaturated fatty acids contain one double bond in the hydrocarbon chain, and polyunsaturated fatty acids contain more than one double bond. Fatty acids in plants usually range from 4- to 26-carbons in size, but oleic acid (18:1) and linoleic acid $(18: 2)$ are the most prevalent in nature Fatty acids are responsible for many of the physical and chemical properties of lipids. Olive oil contains monounsaturated fatty acids which help in lowering LDLcholesterol, while protecting HDL-cholesterol when consumed in moderation in place of saturated fats.

\section{Dietary fiber}

Dietary fiber is defined as of non-digestible carbohydrates and lignin that are intrinsic and intact in plants. The daily recommended intake of fiber is around $20-35 \mathrm{gms}$. Dietary fiber includes a wide array of macromolecules showing a number of physical and chemical properties. The main components included as fiber are cellulose, hemicelluloses, pectins, \& lignin

a. Cellulose: is a cell wall polymer of $\beta-1,4$-linked glucose. In fruits and vegetables, the cell wall constitutes $1 \%$ to $2 \%$ of the fresh weight, and cellulose could be as much as $33 \%$ of that amount.

b. Hemicelluloses: A number of cell wall polymers which are soluble in alkalis are classified as hemicelluloses also known as cross-linking glycan. The primary cell wall constitutes around $30 \%$ of hemicellulose. c. Pectin: $40 \%$ of the total cell wall polysaccharides found in fruit tissues are pectins. Pectins are extracted from certain fruits and vegetables such as citrus, apples and beets for commercial manufacturing of jams and jellies,

d. Lignin: Lignin is a highly resistant polymer present in secondary cell walls, and is associated with fibres and xylem vessels. In the case of fruits and vegetables, lignin content is relatively low.

\section{Vitamins}

Vitamins are important organic molecules required in trace amounts for normal development. Vitamins need to be obtained from diet because living beings cannot make it on their own. The term "vitamin" is derived from the word "vital amine" because the first vitamin discovered was thiamine that contained an amino group. The 14 vitamins known today are vitamin A (retinol), B complex [B1 (thiamine), B2 (riboflavin), B3 (niacin), B5 (pantothenic acid), B6 (pyridoxine), B9 (folate/folic acid), biotin, choline and B12 (cyanocobalamine)] and vitamins $\mathrm{C}, \mathrm{D}, \mathrm{E}$ and $\mathrm{K}$. They are usually grouped into fat-soluble (A, D, E and K) and water-soluble (B group and $C$ ) molecules. The vitamins present in fruits and vegetables make an important contribution to human nutrition, as they have specific functions in normal body performance. The vitamin content of fruits and vegetables shows a wide variation among species.

\section{Vitamin A}

Vitamin A plays an important role in

a. vision,

b. cell division and differentiation,

c. bone development and reproduction

The average daily requirement of vitamin $\mathrm{A}$ for an adult is estimated at 5000 international units. ( $1 \mathrm{IU}=0.3 \mu \mathrm{g}$ retinol or $0.6 \mu \mathrm{g} \beta$ carotene) Carotenoids are lipid soluble pigments responsible for imparting yellow, orange and red colour in fruits and vegetables. Vegetables that provide good amounts of carotene include carrots, pumpkins and squashes. In comparison to vegetables, fruits are not good source of carotenoids, but with a few exceptions include as apricot, mango, citrus, papaya and watermelon. Tomatoes and peppers also contain high levels of carotenoids.

\section{Vitamin B}

Vitamin B complex is a group of water soluble vitamins which includes the following Table 1.

Table I Vitamin B complex

\begin{tabular}{|c|c|c|c|c|}
\hline $\begin{array}{l}\text { Name of } \\
\text { the vitamin }\end{array}$ & Function & Requirement & Sources & Deficiency disease/symptoms \\
\hline Thiamine(BI) & Metabolism of carbohydrates & $\begin{array}{l}\text { I-2mg per day } \\
\text { for a normal } \\
\text { adult }\end{array}$ & Legumes & Beriberi \\
\hline Riboflavin(B2) & $\begin{array}{l}\text { Important part of FAD \& FMN, electron } \\
\text { transfer reactions }\end{array}$ & $\begin{array}{l}\text { I-2mg per day } \\
\text { for a normal } \\
\text { adult }\end{array}$ & $\begin{array}{l}\text { Green vegetables such as beans, } \\
\text { beets, peppers \& spinach are } \\
\text { particularly rich in riboflavin }\end{array}$ & Ariboflavinosis \\
\hline Niacin(B3) & $\begin{array}{l}\text { Precursor to NADH, NAD, NAD and } \\
\text { NADP essential for energy transformation } \\
\text { in different body metabolic reactions }\end{array}$ & $10 \mathrm{mg}$ to $15 \mathrm{mg}$ & Almonds & Pellagra \\
\hline
\end{tabular}


Table Continued...

\begin{tabular}{|c|c|c|c|c|}
\hline $\begin{array}{l}\text { Name of } \\
\text { the vitamin }\end{array}$ & Function & Requirement & Sources & Deficiency disease/symptoms \\
\hline Vitamin B6 & $\begin{array}{l}\text { Cofactor in many transamination, } \\
\text { decarboxylation and deamination reactions }\end{array}$ & $1.3 \mathrm{mg}$ & $\begin{array}{l}\text { Beans, cabbage, cauliflower, } \\
\text { spinach, sweet potatoes, grapes, } \\
\text { prunes, avocados and bananas. }\end{array}$ & $\begin{array}{l}\text { Dermatitis around the eyes, elbows } \\
\text { and mouth, along with soreness of } \\
\text { the mouth and a red tongue }\end{array}$ \\
\hline $\begin{array}{l}\text { Pantothenic } \\
\text { acid }\end{array}$ & Important part of enzyme, Coenzyme A & $5 \mathrm{mg}$ & $\begin{array}{l}\text { Peas, beans, nuts, broccoli, } \\
\text { mushrooms, potatoes and sweet } \\
\text { potatoes }\end{array}$ & $\begin{array}{l}\text { Fatigue, headaches, sleep } \\
\text { disturbance, tingling of hands } \\
\text { and feet and lack of antibody } \\
\text { production }\end{array}$ \\
\hline Biotin & Essential in carboxylation & $30 \mu g$ & Synthesized in the intestinal & Depression, sleeplessness and \\
\hline Folic acid(B9) & $\begin{array}{l}\text { Essential for reproduction and normal } \\
\text { growth }\end{array}$ & $400 \mu g$ & $\begin{array}{l}\text { Spinach, cabbage and other green } \\
\text { vegetables }\end{array}$ & Megaloblastic Anemia \\
\hline Vitamin $\mathrm{B} 12$ & For methylation reactions & $2.4 \mu \mathrm{g}$ & $\begin{array}{l}\text { Does not occur in fruits and } \\
\text { vegetables }\end{array}$ & Pernicious anemia \\
\hline
\end{tabular}

\section{Vitamin C}

Vitamin C also known as ascorbic acid is a water- soluble carbohydrate-derived compound. The daily dietary requirement of vitamin $\mathrm{C}$ for males is $75 \mathrm{mg}$ daily, while for females is $90 \mathrm{mg}$. Fruits, vegetables and juices are excellent sources of vitamin C. Individuals in U.S obtain $90 \%$ of their vitamin C through fruits and vegetables. Rich sources of vitamin $\mathrm{C}$ include green leafy vegetables and tropical species of fruits. Moreover, rosehip, jujube and guava, strawberry, kiwifruit, peppers, and citrus fruit, and spinach, broccoli and cabbage among vegetables are good sources of vitamin $\mathrm{C}$.

\section{Vitamin E}

Vitamin E includes two major groups, tocopherols and tocotrienols that are found in eight different forms (four tocopherols and four tocotrienols). Oily seeds, olives, nuts, peanuts, avocados and almonds contain Vitamin E in abundance. Although tocopherol levels in broccoli and leafy vegetables are less than in fat-rich products, they still they are better sources compared to other fruits and vegetables.

\section{Vitamins $\mathbf{D}$ and $\mathbf{K}$}

Vitamin D comprises of a group of fat-soluble compounds. Two main forms of vitamin D are ergocalciferol and cholecalciferol. In fruits and vegetables, vitamin D is present in negligible amounts. Vitamin $\mathrm{K}$ is known to be important for coagulation of blood. Its RDA is $120 \mu \mathrm{g}$. Vitamin $\mathrm{K}$ is found in abundance in lettuce, spinach, cauliflower and cabbage. Apart from diet, it can also be manufactured by gut bacteria

\section{Minerals}

\section{Potassium (K)}

Potassium is an important mineral found in abundance in fruits and vegetables. Its quantity in fresh tissue varies from $60-600 \mathrm{mg}$ per 100 g. Potassium occurs in combination with variety of organic acids in fruits and vegetables. A diet rich in potassium results in lowering of blood pressure. Potassium helps in regulating heartbeat, improving muscle contraction and sending nerve impulses along with releasing energy from macronutrients including fat, carbohydrates and protein. Potassium is a systemic electrolyte and is essential in co-regulating ATP with sodium. Potassium acts as an acid-base buffer. It also helps in reducing the risk of developing kidney stones, and decreasing bone loss with age. Fruits and vegetables rich in potassium include bananas and plantains, leafy green vegetables, many dried fruits, oranges and orange juice, cantaloupes and honeydew melons, tomatoes and root vegetables.

\section{Calcium (Ca)}

Calcium is essential for bone and tooth formation. Inadequate intake of calcium may increase the risk of osteoporosis. In plants, calcium is primarily associated with the pectic materials. It is believed to have a major influence on the rheological properties of the cell wall and, consequently, on the texture and storage life of fruits and vegetables.

\section{Magnesium (Mg)}

Another important mineral is magnesium responsible for synthesis of protein, release of energy from muscle storage, regulation of body temperature, proper functioning of heart and plays an important role in bone formation. Magnesium is required for activation of over 100 enzymes. In plants, magnesium is a constituent of the chlorophyll molecule: the porphyrin- like ring structure of chlorophylls contains a central magnesium atom coordinated to the four pyrrole rings. An average of $14 \%$ of magnesium in the US food supply is through vegetables in 2004. Generally, magnesium levels in vegetables are significantly greater than in fruits, but nuts are good sources of this nutrient. Dry fruits and legumes have highest magnesium content.

\section{Phosphorus (P)}

Inorganic phosphate is essential for skeletal mineralization and for multiple cellular functions, including glycolysis, gluconeogenesis, DNA synthesis, RNA synthesis, cellular protein phosphorylation, phospholipid synthesis and intracellular regulatory roles. Phosphorus is a primary bone-forming mineral. In 2004, the primary contributor of phosphorus to the food supply was the dairy group (31.3\%), followed by the meat, poultry and fish group $(24.9 \%)$ and grain products (19.4\%). Fruit and vegetable contribution to the total phosphorus in the US food supply was an average of $9.5 \%$. Among tree fruits, nuts are natural sources of phosphorus.

\section{Manganese (Mn)}

Manganese is an important part of enzyme systems, including oxygen-handling enzymes. Key functions include brain function and reproduction and are required for blood sugar regulation. In addition, it is part of bone structure. Manganese is a cofactor in function of antioxidant enzymes; Spinach is a good source of manganese. 


\section{Copper (Cu)}

Copper plays an important role in the oxidative defense system. It is essential for hemoglobin formation and is required for the function of over 30 proteins. In plants, copper is required for synthesis of chlorophyll s and in several copper- containing enzymes involved in the reduction of molecular oxygen. The vegetable group was the leading source of copper (30\%) in the US between 1909-1919.

\section{Iron (Fe)}

Iron is important in synthesis of a number of important proteins, such as the heme-containing proteins, electron transport chain and microsomal electron transport proteins. In green leafy vegetables, there is a good correlation between iron supply and chlorophyll content. Almonds, pistachio nuts, walnuts, pecans, etc. along with different vegetables (e.g. parsley, broccoli, kale, turnip greens and collards) and legumes (e.g. green peas and beans) are considered good sources of iron.

\section{Zinc (Zn)}

Zinc is a pervasive microelement that plays a catalytic or a structural role in more than 200 enzymes such as carboxypeptidase. Zinc also plays a role in the maintenance of cell membrane integrity, and the synthesis of RNA and tryptophan. Fruits and vegetables account for only $1.2 \%$ and $6.4 \%$, respectively, of the zinc in the American food supply. Fruits are poor in zinc, but pecans and walnuts are good sources of this essential mineral. Parsley is also a good source of zinc.

\section{Sodium $(\mathbf{N a})$}

Sodium is a systemic ion important in electrolyte balance and in the regulation of blood pressure It is also essential in co-regulating ATP with potassium. With an increase in intake of processed vegetables, sodium consumption as also raised that has led to development of hypertension among public. Main dietary source for sodium is Table salt $(\mathrm{NaCl})$ along with olives and spinach also being important sources of sodium. Fruits are poor in sodium, and are generally recommended for low-sodium dietary patterns. ${ }^{9}$

\section{Antioxidants in Fruits and Vegetables}

An antioxidant is a compound that helps in stopping cellular oxidation \& prevents body from chronic disease. Fruits and vegetables are rich sources of antioxidant in our diets that helps in reducing the incidence of cardiovascular disease and other degenerative diseases associated with oxidative damage. Antioxidants found in fruits \& vegetables are
a. Ascorbic acid,
b. Carotenoids,
c. Vitamin E and
d. Phenolic compounds including phenolic acids and flavonoids. ${ }^{9}$

\section{Role of fruits \& vegetables in disease prevention}

Fruits and vegetables play a very important role to in preventing from a number of chronic diseases and provide a wide array of beneficial effects on our health. Not a single fruit or vegetable is responsible for good health but their wide variety in terms of colors, flavours \& texture offer great health benefits. Eating recommended amounts can help reducing health hazards. ${ }^{10}$

\section{Obesity}

Obesity is the mother of all diseases. Weight management requires a balance in dietary intake. For this purpose fruit and non-starchy vegetables can play an important role. The high water and fiber content and a relatively low amount of fat and calories, makes fruit and non-starchy vegetables a unique component in reducing weight by increasing satiety and reduce the feeling of hunger. Furthermore, the flavonoids found in fruit and vegetables may ward off obesity through stimulating release of certain hormones that regulate lipid metabolism (such as adiponectin and adipokine), reverse insulin resistance or modulate appetite and energy expenditure. Different researches in adults have shown that consuming more fruit and vegetables could possibly contribute to a stable weight, and probably lead to weight loss if the fruit and vegetables consumed were to replace foods rich in fat or high in energy density. Another study conduct on 74000 women aged 38-63 in the United States (US) found that during a 12-year follow-up, those who were in the quintile with the largest increase in fruit and vegetable consumption had a $24 \%$ lower risk of becoming obese compared with those who were in the quintile with the largest decrease in consumption. ${ }^{11}$

\section{Cardiovascular diseases (hypertension, coronary heart disease and stroke.)}

Many research studies has evidently showed that a diet rich in plant products like fruits and vegetables is good enough in lowering the risk of CVD's (hypertension, coronary heart disease (CHD) and stroke). A cohort study of over 28000 middle-aged and older women in the U.S. with a mean of 12.9 years of follow-up has found an inverse dose-response relationship between total fruit and vegetable consumption and the risk of hypertension compared with the women who consumed less than 2 servings of fruit and vegetables a day, women who consumed 2 to less than 4 servings, 4 to less than 6 servings, 6 to less than 8 servings, and 8 or more servings of total fruit and vegetables a day had a $3 \%, 7 \%, 11 \%$, and $14 \%$ lower risk of hypertension respectively. For CHD, a meta-analysis of 13 independent cohort studies indicated that individuals who had 3 to 5 servings and more than 5 servings of fruit and vegetables a day had a respective $7 \%$ and $17 \%$ lower risk of CHD when compared with those who had less than 3 servings of fruit and vegetables a day. Similarly, pooled analyses of cohort studies reported that people who consumed fruit and vegetables 3 to 5 servings and more than 5 servings a day had a respective $11 \%$ and $26 \%$ reduced risk of stroke when compared with those who consumed less than 3 servings a day. ${ }^{12}$

\section{Cancer}

According to World Cancer Research Fund (WCRF) and the American Institute for Cancer Research (AICR) a diet that provides more than $400 \mathrm{~g}$ of fruit and vegetables a day can reduce the risk of at least $20 \%$ of all cancer incidences. Cancers of the mouth, pharynx, larynx, oesophagus, and stomach can be prevented by taking a diet rich in fruits and vegetables. For example, a meta-analysis of 16 studies that examined the effects of fruit and vegetable consumption on the occurrence of oral cancer reported that each serving of fruit and each serving of vegetables consumed per day significantly lowered the risk of oral cancer by $49 \%$ and $50 \%$ respectively. Pooled analyses of case-control studies showed that for each $100 \mathrm{~g}$ of fruit consumed per day, there were respectively $27 \%$ reduced risk of laryngeal cancer and $28 \%$ reduced risk of oesophageal cancer; for each $100 \mathrm{~g}$ of vegetables consumed per day, there was $11 \%$ lower risk of esophageal 
cancer. Allium vegetables (such as garlic, leeks, onions, shallots or chives) are helpful in protecting against cancer of the stomach and colorectal in particular. A pooled analysis of 21 studies reported that there is $46 \%$ reduction in gastric cancer risk in individuals with the highest consumption of allium vegetables than to those with the lower intake. ${ }^{13}$ Another meta-analysis of 14 cohort studies reported that per $80 \mathrm{~g}$ serving of fruit per day was associated with a $6 \%$ lower risk of lung cancer. Consuming fruits and vegetables with high lycopene content like tomatoes helps in lowering risk of prostate cancer in men along with providing general ability to fight and prevent cancer risk. Bladder cancer risk can be combated by an increased intake of cruciferous vegetables, such as cauliflower, broccoli and cabbage. A meta-analysis of 10 cohort and case-control studies observed a significant $20 \%$ reduced risk of bladder cancer in the group with the highest cruciferous vegetables consumption compared with the group with the lowest consumption. ${ }^{14}$

\section{Type 2 diabetes}

Most of the epidemiological studies showed no significant risk relation between diabetes and consumption of fruit and vegetables as a whole, as well as consumption of either fruit or vegetables alone. However, one meta-analysis released in 2010 of six prospective cohort studies with over 220000 participants aged 30-74years showed that the risk of type 2 diabetes was significantly lower in persons who took relatively large amount of green leafy vegetables..$^{15}$

\section{Chronic respiratory diseases (asthma and chronic obstructive pulmonary disease)}

A great number of epidemiological and clinical evidence reported a relation between higher intake of fruit and vegetables and a lower risk of asthma or asthma-related symptoms as well as chronic obstructive pulmonary disease (COPD). A prospective birth cohort study that investigated associations between childhood diet and asthma outcomes at 8years of age reported that one day per week of fresh fruit consumption at early age was associated with a $7 \%$ reduced risk of asthma symptoms; long-term consumption of fresh fruit from 2 to 8years of age was also associated with a $10 \%$ reduction in risk. Similarly, pooled analyses of 4 cross- sectional studies showed that high consumption of fruit (but not vegetables) by children aged $10-14$ years was associated with a $25 \%$ reduced risk of wheezing. In addition, one prospective study of over 68000 adult women observed that those in the highest quartile of carrot, leafy vegetable and tomato consumption had a 19\%, 18\% and $15 \%$ reduced risk of asthma respectively as compared to their peers in the lowest quartile. ${ }^{16}$

\section{Cognitive decline or dementia}

Different research studied supported that taking a diet rich in fruit and vegetable might protect against age-related cognitive decline or dementia. A systematic review of 9 cohort studies with a total of around 44,000 participants found that frequent intake of vegetables, but not fruit, was associated with slower rates of cognitive decline and a lower risk of dementia in older age. Studies also found that better cognitive function was associated with high consumption of certain subgroups of fruit and vegetables, including citrus fruit, berry fruit, green leafy vegetables, cruciferous vegetables, legumes, carrots, or vitamin C-rich fruit and vegetables. ${ }^{17}$

\section{Eye diseases (macular degeneration and cataract)}

Some research studies suggested that generous intake of fruit and green leafy or cruciferous vegetables rich in carotenoids, lutein and zeaxanthin might lower the risk of age-related macular degeneration (AMD) or age-related nuclear cataract. One prospective study that followed over 110,000 men and women aged 50 and above for about 18 years showed that people who took 3 or more servings of fruit daily had a $36 \%$ reduced risk of AMD compared to those who consumed less than 1.5 servings per day. No significant protective effect was observed for vegetables. ${ }^{18}$

\section{Rheumatoid arthritis}

Systematic reviews of literature on the role of fruit and vegetables in susceptibility to rheumatoid arthritis found that high intake of fruit and vegetables possibly cut rheumatoid arthritis risk or relieve inflammatory rheumatoid symptoms. To examine any relationship between intake of fruit and vegetables and the risk of inflammatory polyarthritis like rheumatoid arthritis, a study followed over 25,000 men and women aged 45-74years for 9years and analyzed their dietary patterns. This study suggested that individuals whose intake were lowest regarding fruit and vegetables combined (less than $167 \mathrm{~g}$ a day), fruit (78g or less a day), and vegetables (less than $64.6 \mathrm{~g}$ a day) had a respective $90 \%, 60 \%$, and $30 \%$ risk of developing inflammatory polyarthritis than those who consumed the highest amounts of fruit and vegetables combined (more than $275 \mathrm{~g}$ a day), fruit (more than $176 \mathrm{~g}$ a day), and vegetables (more than $113 \mathrm{~g}$ a day). In addition, a case control study also showed that increased consumption of cooked vegetables might reduce the risk of rheumatoid arthritis. ${ }^{17,19}$

\section{Fruits \& vegetables - the actual fast foods}

Throughout the world, the load of NCD's is increasing rapidly. $60 \%$ of 56million deaths globally in 2001 were attributed to noncommunicable diseases. The leading factors in increase of NCD's include increased cholesterol levels in blood, less consumption of fruits and vegetables, high intake of energy dense foods, BMI greater than 30 , sedentary lifestyle. ${ }^{20}$ Now-a-days what people consider as fast food includes energy dense foods instead of nutrient rich foods. Energy dense foods are foods high in fat, salt, sugar \& refined products such as burgers, pizzas, carbonated beverages $\&$ lots more, whereas nutrient dense foods include fruits \& vegetables. What actually needs to be done is to make shift of people's perception about fast foods from energy dense foods to nutrient rich foods. Fruits \& vegetables should be called the original fast foods. Fruits and vegetables are truly lifesaving. According to World Health Organization sufficient fruit and vegetable consumption is required to save up to 2.7 million lives annually. In another report presented by World Health in 2002, low fruit and vegetable consumption is among the top ten risk factors responsible to early mortality. ${ }^{20}$

Flavonoids responsible for health impart colors to fruits and vegetables. Different colors represent different nutrients and benefits. For example, purple, red, and blue fruits or vegetables are rich in phytochemicals called "anthocyanin," which help get rid of free-radical damage in the body and the brain. Orange, yellow, and green vegetables are rich in stress-lowering carotenoids. According to a research some of these carotenoids, such as beta-carotene, when consumed on a regular basis, actually have a stress hormone-lowering effect, so enjoy eating colourful rainbow of fruits and vegetables in everyday life. ${ }^{21}$

\section{Global review on fruit \& vegetable campaigns}

From last two decade, due to growing awareness regarding benefits of fruits and vegetables for health there is an immense increase in campaigns for these plant products around the globe. 
Two major supporters of such campaigns are WHO and FAO. Both of these organizations conducted a conference namely" Joint FAO/ WHO Workshop on Fruit and Vegetables for Health" in Kobe, Japan in year 2004. The purpose of this conference was to bring together the evidence and develop a draft framework to guide the development of cost-efficient and effective interventions to promote adequate consumption of fruit and vegetables in Member States. ${ }^{22} \mathrm{~A}$ key global advocate for the development of these campaigns is the International Fruit and Vegetable Alliance (IFAVA), an organization with a membership of national campaigns groups and an internal board of directors. The aim of IFAVA is to encourage and foster efforts to increase the intake of fruit and vegetables worldwide for better health by supporting national initiatives, promoting efficiencies, facilitating collaboration on shared aims and providing global leadership. At the national level, campaigns typically promote the consumption of a certain number of fruits and vegetables a day - such as " 5 a day" or "6 a day". Such campaigns aim to encourage general populations to increase their consumption of fruits and vegetables.

In US, the original "5 a day" program began again in 2007 under the brand name of "More Matters" in order to make the message more compelling. In Western Australia, the approach to promoting fruits and vegetables has evolved on the basis of ongoing evaluations. The first campaign began in early 1990s intended to provide information regarding importance of consuming more fruits and vegetables. This campaign helped in increasing awareness among general population but no change was noticed among consumer behavior. The second campaign which started in 2005 ("Go for $2 \& 5$ ") provided more specific information on the quantity that is required by people to consume, but again, no behavioral changes were seen except the awareness. The campaign was then refined to include more "provocative" messages, which was attributed with boosting vegetable consumption. ${ }^{23}$

\section{Conclusion}

Globally, the burden of non-communicable diseases has rapidly increased. In year 2001 non- communicable disease accounted for 60 percent of the 56million deaths worldwide and 47 percent of the global burden of disease. Underlying determinants for noncommunicable diseases includes increased consumption of energydense, nutrient-poor foods that are high in fat, sugar and salt; reduced levels of physical activity: and of particular concern are the increasingly unhealthy diets and reduced physical activity of children and adolescents. Cardiovascular diseases and cancers are main causes of morbidity and mortality in the UK, with cancer responsible for 127,000 deaths every year and coronary heart disease killing a further 110,000 a year. Although death rates have decreased slowly every year since 1985, these diseases continue to be main causes of mortality in the UK. Decreasing the prevalence of both diseases is therefore still an important goal in the public health strategy. ${ }^{24,25}$

A high consumption of fruit and vegetables is generally considered to be beneficial to health, and there is now a comprehensive body of evidence recognizing the protective effects of high fruit and vegetable consumption on cardiovascular disease and certain cancers, particularly epithelial cancers. Dietary advice to the public encouraging increased consumption of fruit and vegetables has been promoted since the 1970 s, owing to the great nutritional quality \& health benefits provided by them in reducing non-communicable diseases. The nutrients obtained from fruits and vegetables include water, carbohydrates, fats, proteins, fiber, minerals, organic acids, pigments, vitamins and antioxidants, among Others. Most fruits and vegetables are available almost year-round in a wide variety and they not only taste good, but they also have favorable attributes of texture, colour, flavour and ease of use.

Fruits \& vegetables should be called as fast food. They can be consumed fresh, cooked, hot or cold, canned, pickled, frozen or dried. Fruits and vegetables are consumed at all times, and due to their convenient size; they are an excellent between-meal snack. They are relatively low in calories and fat (avocado and olives being the exceptions), they have no cholesterol, they are rich in carbohydrates and fiber, they contain vitamin $\mathrm{C}$ and carotene, and some are a good source of vitamin B complex. Fruits and vegetables are relatively low in sodium and high in potassium. Ascorbic acid in fruits and vegetables enhances the bioavailability of iron in the diet. Because of all these characteristics, fruits and vegetables have a unique role in a healthy diet with the increasing recognition that fruit and vegetables are beneficial to health, official recommendations have been made as to the desirable quantities of fruit and vegetables that people should consume. The World Health Organization (WHO) recommended an intake of at least $400 \mathrm{~g}$ of fruit and vegetables, per person per day, to provide protection against chronic diseases. Recommendations simply state that five portions of fruit and vegetables per day should be taken including a diverse range of fruit and vegetables should be included in the daily diet. This diversity is important because different fruit and vegetables contain different types and amounts of nutrients and phytochemicals, and therefore a greater diversity of foods will lead to a greater diversity of nutrient and phytochemical intakes.

\section{Acknowledgements}

None.

\section{Conflict of interest}

The author declares no conflict of interest.

\section{References}

1. Ashakiran, Deepthi. Fast Foods and their Impact on Health. JKIMSU. 2012;1(2):7-14.

2. Wang L, Manson JE, Gaziano JM, et al. Fruit and vegetable intake and the risk of hypertension in middle-aged and older women. Am J Hypertens. 2012;25(2):180-189.

3. Valeria Menza, Claudia Probart. The Eating well for good health. 2013.

4. Wojciech J Florkowski, Robert L Shewfelt, Bernhard Brueckner, et al. Postharvest Handling: A Systems Approach. 2nd ed. USA: Elsevier Inc, Academic Press; 2009.

5. Pattison DJ, Harrison RA, Symmons DP. The role of diet in susceptibility to rheumatoid arthritis: a systematic review. J Rheumatol. 2004;31(7):1310-1319.

6. Hyson, Dianne. The Health Benefits of Fruits and Vegetables: Scientific Overview for Health Professionals. Review of literature from 1999 to 2001. Produce for Better Health Foundation. 2002.

7. Joceline. Effectivness of interventions \& programs promoting fruits \& vegetables intake. 2004.

8. Center of health protection. Non communicable diseases. Department of Health, 2013.

9. Riboli E, Norat T. Epidemiologic evidence of the protective effect of fruit and vegetables on cancer risk. Am J Clin Nutr. 2003;78(3 Suppl):559S$569 \mathrm{~S}$. 
10. Hughes LA, Arts IC, Ambergen T, et al. Higher dietary flavone, flavonol, and catechin intakes are associated with less of an increase in BMI over time in women: a longitudinal analysis from the Netherlands Cohort Study. Am J Clin Nutr. 2008;88(5):1341-1352.

11. He $\mathrm{K}, \mathrm{Hu} \mathrm{FB}$, Colditz GA, et al. Changes in intake of fruits and vegetables in relation to risk of obesity and weight gain among middleaged women. Int J Obes Relat Metab Disord. 2004;28(12):1569-1574.

12. Willers SM, Wijga AH, Brunekreef B. Childhood diet and asthma and atopy at 8 years of age: the PIAMA birth cohort study. Eur Respir J. 2011;37(5):1060-1067.

13. Liu B, Mao Q, Lin Y, et al. The association of cruciferous vegetables intake and risk of bladder cancer: a meta-analysis. World J Urol Lutein \& Zeaxanthin. 2012;31(1):127-133.

14. Rui Hai Liu. Health benefits of fruit and vegetables are from additive and synergistic combinations of phytochemicals. Am Soc Clin Nutr. 2013;78(3 Suppl):517S-520S

15. Carter P, Gray LJ, Troughton J, et al. Fruit and vegetable intake and incidence of type 2 diabetes mellitus: systematic review and metaanalysis. BMJ. 2010;341:C4229.

16. World Health Report. Reducing Risks, Promoting Healthy Lives. 2002.

17. Pennington JAT, Fisher RA. Classification of fruits and vegetables. $J$ Food Comp Anal. 2009;22:23S-31S.
18. Olga Vea. Consumption of fruit and vegetables and associated factors among 11 to 13 year old children in Portugal. 2007.

19. Pattison DJ, Silman AJ, Goodson NJ, et al. Vitamin C and the risk of developing inflammatory polyarthritis: prospective nested case-control study. Ann Rheum Dis. 2004;63(7):843-847.

20. Oyedunni S Arulogun, Modupe O Owolabi. Fast Food Consumption Pattern among Undergraduates of the University of Ibadan, Nigeria: Implications for Nutrition Education. J Agric Food Tech. 2011;1(6):8993.

21. WHO and FAO. Fruit and Vegetable Promotion Initiative. 2003.

22. Boyer J, Liu R. Apple Phytochemicals and their health benefits. Nutr J. 2004;3:5.

23. Hawkes C. Promoting healthy diets through nutrition education \& changes in food environment, an international review of actions \& their effectiveness. 2013.

24. Centers for Disease Control and Prevention. Strategies to Prevent Obesity and Other Chronic Diseases: The CDC Guide to Strategies to Increase the Consumption of Fruits and Vegetables. USA: US Department of Health and Human Services; 2011.

25. Loef $M$, Walach $H$. Fruit, vegetables and prevention of cognitive decline or dementia: a systematic review of cohort studies. J Nutr Health Aging. $2012 ; 16(7): 626-630$ 